\title{
HemoCheck: um aplicativo para pacientes com Hemofilia
}

\author{
HemoCheck: an application to patients with Hemophilia \\ HemoCheck: una aplicación para pacientes con Hemofilia
}

Recebido: 07/03/2021 | Revisado: 12/03/2021 | Aceito: 15/03/2021 | Publicado: 22/03/2021

\author{
Luís Guilherme Cordeiro Santos Silva \\ ORCID: https://orcid.org/0000-0003-3211-5411 \\ Universidade de Rio Verde, Brasil \\ E-mail: luisguilhermecordeiro@gmail.com \\ Isabella Aparecida Vasconcelos Moreira \\ ORCID: https://orcid.org/0000-0001-6172-561X \\ Universidade de Rio Verde, Brasil \\ E-mail: isabellaavm133@ gmail.com \\ Berenice Moreira \\ ORCID: https://orcid.org/0000-0003-4077-8952 \\ Universidade de Rio Verde, Brasil \\ E-mail: berenice@unirv.edu.br
}

\begin{abstract}
Resumo
O tratamento da hemofilia realizado em domicílio apresenta a necessidade de controle das infusões, feito por meio do registro em um diário impresso. Objetivo: descrever o processo de desenvolvimento de um aplicativo móvel de follow up para hemofílicos. Metodologia: O desenvolvimento do aplicativo ocorreu em duas etapas, sendo a primeira correspondente à análise da demanda dos hemofílicos e a segunda se trata do design e desenvolvimento, criado a partir de um mesmo código, utilizando uma tecnologia híbrida. Resultados: O HemoCheck está disponível nas plataformas Android e iOS; oferta telas que fornecem orientações sobre a doença, para o registro do histórico de infusões e intercorrências; para o perfil do usuário e um botão para contatar o hemocentro cadastrado. Conclusão: O estudo cumpriu o objetivo proposto e diante dos resultados encontrados, recomenda-se que seja feito a análise de qualidade do HemoCheck e o impacto do mesmo na vida dos pacientes por meio de outras pesquisas.
\end{abstract}

Palavras-chave: Software de computador; Algoritmos; Análises de dados; Hemofilia A; Hemofilia B.

\begin{abstract}
The treatment of hemophilia carried out at home presents the need to control infusions, made through the registration in a printed diary. Objective: To describe the process of developing a follow up mobile application for patients with hemophilia. Methodology: The development of the application took place in two stages, the first corresponding to the analysis of the demand of hemophiliacs and the second it is about design and development, created from the same code, using a hybrid technology. Results: HemoCheck is available on Android and iOS platforms; offer screens that provide guidance on the disease, for recording the history of infusions and complications; to the user's profile and a button to contact the registered blood center. Conclusion: The study fulfilled the proposed objective and given the results found, it is recommended that the quality analysis of HemoCheck and its impact on patients' lives be carried out through other inquiries.
\end{abstract}

Keywords: Computer software; Algorithms; Data analysis; Hemophilia A; Hemophilia B.

\section{Resumen}

El tratamiento de la hemofilia realizado en el domicilio presenta la necesidad de controlar las infusiones, realizadas mediante el registro en un diario impreso. Objetivo: describir el proceso de desarrollo de una aplicación móvil de seguimiento para hemofílicos. Metodología: El desarrollo de la aplicación se realizó en dos etapas, la primera correspondiente al análisis de la demanda de hemofílicos y la segunda se trata de diseño y desarrollo, creado a partir de un mismo código, utilizando una tecnología híbrida. Resultados: HemoCheck está disponible en las plataformas Android e iOS; ofrecer pantallas que brinden orientación sobre la enfermedad, para registrar el historial de infusiones y complicaciones; al perfil del usuario y un botón para contactar con el centro de sangre registrado. Conclusión: El estudio cumplió con el objetivo propuesto y dados los resultados encontrados, se recomienda que se realice el análisis de calidad de HemoCheck y su impacto en la vida de los pacientes a través de otras investigaciones.

Palabras clave: Software de ordenador; Algoritmos; Análisis de los datos; Hemofilia A; Hemofilia B.

\section{Introdução}

A coagulação sanguínea consiste em uma série de reações químicas que convertem o fibrinogênio em fibrina, por 
meio da enzima trombina, quando ocorrem lesões vasculares. Os fatores de coagulação I a XIII são pró-enzimas e enzimas, fundamentais na formação da trombina. No final da cascata de coagulação a fibrina é liberada pelo fator XIIIa, posteriormente, forma fibras que consolidam o tampão plaquetário e o transforma em tampão hemostático. Deste modo, impede que o sangramento evolua (Cagnolati, Sankarankutty, Rocha, Beer, \& Silva, 2017).

A hemofilia é um distúrbio hemorrágico, genético e hereditário associado ao cromossomo X caracterizada pela deficiência ou disfunção dos fatores de coagulação. A hemofilia A (HA) ou hemofilia clássica afeta o fator VIII, enquanto a hemofilia B (HB) ou Doença de Christmas incide sobre o fator IX, sendo transmitida devido a uma herança recessiva ligada ao sexo, cuja mãe portadora da mutação a transfere para o filho, representando $70 \%$ dos casos. Contudo, o desenvolvimento pode acontecer em virtude de uma mutação denominada "de novo" ou esporádica, acometendo indivíduos sem histórico familiar em 30\% dos casos (Brasil, 2011; Brasil, 2015).

A HA está presente em cerca de um a cada 5.000-10.000 nascidos vivos do sexo masculino. Em contrapartida, aproximadamente um a cada 40.000-50.000 nascidos vivos do sexo masculino, são hemofílicos B, sendo a HA prevalente e correspondendo a $80 \%$ dos pacientes. Em 2016, no Brasil, o número de pacientes com coagulopatias era 24.228, destes 10.123 $(41,78 \%)$ eram portadores de HA e $1.996(8,24 \%)$ de HB. A região que mais concentra hemofílicos é a Sudeste $(46,21 \%)$, isso porque a região é mais populosa. A faixa etária predominante é entre 20 e 29 anos. Ressalta-se que existem casos não notificados ou registrados inadequadamente no sistema Hemovida Web Coagulopatias, portanto, estes números podem ser maiores (Barca et al., 2010; Brasil, 2018; WFH, 2012).

Quanto à gravidade, esse distúrbio é classificado segundo os níveis plasmáticos do fator de coagulação, da seguinte maneira: grave, <1\%; moderada, entre 1-5\%; e leve, entre 5-40\%. Hemofílicos graves geralmente são diagnosticados quando ainda são bebês, pois podem apresentar sangramentos espontâneos, divergindo dos pacientes com hemofilia leve ou moderada, porque estes apresentam hemorragias apenas quando ocorre um trauma ou são submetidos a uma cirurgia, dificultando o diagnóstico (WFH, 2012).

As hemofilias possuem manifestações clínicas semelhantes. Os principais sinais e sintomas são: hemartroses, hemorragias, epistaxe, hematúria, melena, hematomas, entre outros. Geralmente, ocorrem após um trauma de intensidade mínima, exceto no caso de hemofílicos graves, no qual o sangramento pode se manifestar espontaneamente. Quando o nível plasmático do fator é superior a 40\%, não existem hemorragias (Brasil, 2011; Zazo, Falcão \& Pasquini, 2013).

Em relação ao diagnóstico, primeiramente, são realizados os seguintes testes de triagem: Tempo de Protrombina (TP), o Tempo de Tromboplastina Parcial Ativado (TTPA) e a dosagem dos fatores VIII e IX. Após estes exames laboratoriais, é importante verificar a presença de inibidores neutralizantes da função do fator, anticorpos que dificultam o controle de sangramentos e a terapia do paciente, por meio do método quantitativo de inibidor (Brasil, 2016).

Existem duas modalidades de tratamento: sob demanda e profilático. O tratamento sob demanda compreende a reposição do fator de coagulação deficiente de origem plasmática ou recombinante por via endovenosa, após um episódio hemorrágico. A administração é diária até a sintomatologia cessar. Já o tratamento profilático, possui 3 subtipos: primário, secundário e terciário. Nos quadros com inibidores, é realizada a Imunotolerância-IT, em que as doses do fator deficiente são administradas diariamente ou em dias alternados, com a finalidade de dessensibilizar o indivíduo. Assim que os inibidores cessam, o paciente retorna ao tratamento habitual (Portaria no 364, 2014; Portaria $n^{\circ} 478,2014$ ).

Todas as infusões podem ser realizadas em ambiente hospitalar, ambulatorial e/ou domiciliar. A administração em residências deve ser feita somente pelo familiar ou profissional de saúde treinados adequadamente pelo Centro de Tratamento de Hemofilia - CTH. Essa possibilidade reduz o deslocamento até o centro de referência do paciente, melhora a qualidade de vida, proporciona a autonomia aos familiares e permite a infusão precoce do fator na presença de hemorragias. Para que isto ocorra, os cuidados a seguir precisam ser tomados: um local correto para o armazenamento dos fatores e materiais para 
punções, recipiente para descarte de itens perfuro cortantes e preenchimento do Diário de Infusão (Brasil, 2014; FBH, 2020).

O Diário de Infusão é uma caderneta de registro que permite acompanhar a evolução do paciente, as intercorrências, as infusões de fator, o motivo da aplicação, a dosagem, a data, o horário e o local da administração. Além disto, este diário possui orientações úteis ao pacientes, cuidadores e profissionais de saúde (Pôncio, 2018). Geralmente, os hemofílicos possuem uma versão impressa que é bastante utilizada pelos médicos hematologistas responsáveis pelo tratamento. A partir das informações registradas, consegue-se analisar a evolução do indivíduo e prescrever terapias adequadas.

Com o decorrer dos avanços tecnológicos, o aparelho celular ganhou novas utilidades mediante a ampliação de suas funções. Suas diversas ferramentas aliadas com possibilidade de levá-lo a qualquer lugar, tornaram os smartphones os principais meios de acesso à Internet (Oliveira \& Alencar, 2017).

Segundo a Pesquisa Nacional por Amostra de Domicílios Contínua - PNAD Contínua, em 2016, o telefone móvel celular estava presente em cerca de $92 \%$ dos domicílios, sendo 94,5\% em áreas urbanas e 80,3\% em áreas rurais do Brasil. Na atualidade, é o dispositivo mais utilizado para acessar a internet em 46.735 domicílios, seguido do microcomputador (27.793) e do tablet (8.543). Em 2017, 98,7\% dos domicílios utilizavam o celular com o propósito de acessar a internet, esse percentual aumentou para 99,2\% em 2018 (IBGE, 2016; IBGE, 2018).

Com o aumento da utilização de smartphones e acesso a tecnologias da informação e comunicação é notável o interesse e o crescente número de downloads nas plataformas para aplicativos (apps) de diversas áreas. Isso se deve em boa parte à facilidade com que esses apps podem ser acessados em suas respectivas lojas virtuais (Tibes, Dias \& ZemMascarenhas, 2014).

Pesquisadores e desenvolvedores da área da saúde estão avaliando e utilizando apps para muitos propósitos, como, por exemplo, triagem, monitoramento e autogestão da depressão com resultados positivos para o tratamento da doença (Bindhin, Freeman \& Trevena, 2014); melhoria na adesão ao tratamento por pacientes que se encontram em reabilitação contra o tabagismo (Free et al., 2013); uso da tecnologia móvel para a recuperação em casos de acidente vascular cerebral (Im et al., 2013); controle de exposição a pessoas infectadas com a Covid-19, entre outros.

Esse uso de tecnologias móveis e sem fio auxilia a assistência de saúde em nível mundial. A saúde móvel (mHealth) é definida como prática médica e de saúde pública apoiada por dispositivos móveis, como telefones celulares, dispositivos de monitoramento, assistentes digitais pessoais (PDAs) e outros dispositivos sem fio (WHO, 2011).

A educação em saúde objetiva ampliar a autonomia e a capacidade de intervenção das pessoas sobre suas próprias vidas e as Tecnologias da Informação e Comunicação (TIC) têm o potencial de contribuir de forma importante para melhorar o acesso a serviços de qualidade e, ao mesmo tempo, reduzir custos (Mendez, Salum, Junkes, Almonte, \& Mendez, 2019). As TIC's foram utilizadas na criação de um aplicativo móvel que possibilita a Sistematização da Assistência de Enfermagem SAE, para pacientes com problemas renais, esta tecnologia contribui para a distribuição de serviços na área da saúde (Oliveira et al., 2021).

Nessa perspectiva, o estudo tem como questão norteadora: por que não existe um aplicativo móvel educativo simples, seguro e acessível para registrar os dados importantes dos pacientes com hemofilia e acompanhá-los? Nessa perspectiva, este estudo tem como objetivo descrever o desenvolvimento de um aplicativo móvel educativo de follow up de enfermagem para pessoas com diagnóstico de Hemofilia, visando criar um registro que permite acompanhar a evolução do paciente, as intercorrências, as infusões de fator, o motivo da aplicação, a dosagem, a data, o horário e o local da administração, a fim de facilitar a organização das informações de infusões de fator e intercorrências, além de ofertar orientações básicas de como proceder diante de certas situações. 


\section{Metodologia}

Trate-se de uma pesquisa de desenvolvimento experimental de um aplicativo móvel, com abordagem qualitativa. O estudo de natureza qualitativa requer que haja a interpretação do pesquisador e a exposição da sua opinião crítica sobre o fenômeno em estudo (Pereira, Shitsuka, Parreira, \& Shitsuka, 2018).

O trabalho foi realizado no período de junho de 2020 a janeiro de 2021, e, tem-se como público-alvo os pacientes diagnosticados com hemofilia que fazem o tratamento com a dose domiciliar.

O desenvolvimento experimental consiste em trabalhos sistemáticos baseados nos conhecimentos existentes obtidos por pesquisa ou experiência prática, visando a criação de inovação tecnológica para gerar novos materiais, produtos ou dispositivos, e assim, estabelecer novos processos, sistemas e serviços ou melhorar consideravelmente os já existentes por meio do conhecimento científico (OCDE, 2002; Fuck \& Vilha, 2012).

$\mathrm{O}$ aplicativo foi desenvolvido em duas etapas, a primeira compreende a análise, enquanto a segunda descreve sobre o design e o desenvolvimento, estão dispostos a seguir:

Etapa I - análise: nesta etapa foi realizada a identificação e enumeração das necessidades de orientações dos pacientes com Hemofilia, na qual foi possível constatar as necessidades desses pacientes em relação às dificuldades e dúvidas. Realizouse uma revisão integrativa da literatura que identificou as evidências de tratamentos propostos atualmente para evitar a progressão da doença e proporcionar melhor qualidade de vida. E, por fim, a prospecção tecnológica de similares que foi realizada através da análise de aplicativos (apps) similares já existentes nas lojas virtuais. Na análise, buscou-se descrever a situação atual na produção intelectual voltada à educação em saúde e o acompanhamento em domicílio.

Etapa II: denominada design e desenvolvimento, representada pela elaboração do conteúdo instrucional contextualizado e pela metodologia de desenvolvimento do app educativo para acompanhamento dos pacientes com diagnóstico de Hemofilia. Esta etapa foi organizada em dois momentos, descritos a seguir:

$1^{\circ}$ Momento - Design: período de definição do conteúdo de aprendizado, da estrutura de navegação de telas, assim como a organização visual, funcional e sua tipografia, ou seja, a composição do layout juntamente com questões de percepção, tal como tipo de letra, tamanho de fonte, espaçamento, cores e posicionamento das imagens, figuras e animações. Para tal atividade, houve um profissional da área de tecnologia da informação voluntário que prestou consultoria/apoio nesse momento.

Essa fase foi desenvolvida a partir dos dados obtidos na etapa I. O conteúdo pedagógico foi composto por conceitos da doença, informações sobre fatores de risco, diagnóstico, tratamento e orientação para a prevenção de complicações.

Definiu-se também a linguagem necessária para a efetiva compreensão das informações oferecidas no app. Para a construção dos protótipos de alta fidelidade, utilizou-se a ferramenta Adobe XD, a qual permite a diagramação das telas e seu fluxo de navegação para as plataformas Android e iOS.

Os protótipos de alta fidelidade são basicamente desenhos de telas (esboços) que já apresentam maior semelhança com o design final em termos de detalhes e funcionalidade.

$2^{\circ}$ Momento - A fase de desenvolvimento constituiu a produção do objeto de aprendizagem propriamente dito, ou seja, a codificação do aplicativo em linguagem computacional e armazenamento na plataforma escolhida. Foi realizado por um programador pleno voluntário, o qual utilizou a tecnologia híbrida que atende a múltiplas plataformas. As tecnologias que foram utilizadas e o motivo estão melhores descritos no tópico 2.2 deste trabalho.

\subsection{Arquitetura de Software}

De forma a organizar e estruturar melhor o projeto, durante a fase de desenvolvimento, foi utilizada a arquitetura Model View Controller (MVC), uma vez que ele tem demonstrado seus benefícios por aplicações interativas que permitem múltiplas representações da mesma informação, promovendo a reutilização do código e ajudando os desenvolvedores a se 
concentrarem em um único aspecto da aplicação (Sanders \& Cumaranatunge, 2007).

Esta arquitetura divide o sistema interativo em três componentes, cada um deles especializado em uma tarefa. O modelo contém os dados do aplicativo e gerencia a exibição visual do modelo e o feedback para o usuário. O controlador interpreta as entradas de mouse e o teclado do usuário, comandando o modelo e a visualização para alterar adequadamente (Burbeck, 1992).

\subsection{Linguagens, Bibliotecas e Frameworks}

Para o desenvolvimento do aplicativo, foram utilizadas as seguintes ferramentas e linguagens: Android Studio, Flutter e Visual Studio Code.

\subsubsection{Android Studio}

Para o debug do aplicativo, em caso de erros, foi escolhida a Integrated Development Environment (IDE) Android Studio, pelo fato de ser um ambiente oficial do Google, com isto se torna mais fácil de utilizar os plugins e configurações necessárias para os testes (Google Developers, 2020).

\subsubsection{Flutter}

Para o desenvolvimento deste projeto, será utilizado framework Flutter que foi desenvolvido e é mantido pela Google. O motivo pelo qual este framework foi escolhido se deve à produtividade, uma vez que ele é codificado em cima da linguagem Dart que fornece otimizada compilação para obter um desempenho previsivelmente alto e uma inicialização rápida, portabilidade, devido ao fato de compilar diretamente para ARM e acessibilidade, pois a linguagem Dart é similar a várias outras linguagens, graças a sua orientação a objeto e sintaxe (Flutter, 2020).

Além dos motivos acima citados, também é necessário citar o fato de que o desempenho nos celulares é maior, pois ele suporta a compilação Just-in-time (JIT) e a Ahead-of-time (AOT).

A compilação JIT permite que o Flutter compile novamente o código, enquanto o aplicativo ainda está em execução e isso faz com que a aplicação não perca o estado de desenvolvimento, fazendo, desta forma, um desenvolvimento muito rápido e produtivo.

Já na compilação AOT, as bibliotecas e funções são compiladas em código ARM nativo de cada plataforma, seja essa iOS ou Android.

\subsubsection{Visual Studio Code}

A ferramenta que possibilitará que seja feito o desenvolvimento da aplicação é o Visual Studio Code, uma ferramenta gratuita disponibilizada pela Microsoft que fornece todos os plugins necessários para que façamos proveito das funcionalidades que o Flutter oferece, além de fornecer plugins que irão aumentar e otimizar a velocidade de desenvolvimento da aplicação, como, por exemplo, plugins de auto complete de código, plugins de indentação de código (Visual Studio Code, 2020).

Para a execução deste projeto não foi necessário persistir os dados dentro do próprio aplicativo, uma vez que quem será responsável por manter os dados é a Interface de Programação de Aplicativos (API), sendo esta um conjunto de rotinas e padrões de programação para acesso a um aplicativo de software ou plataforma baseado na Web.

\section{Resultados}

O aplicativo "HemoCheck", nome atribuído à caderneta de infusão virtual, foi desenvolvido para a plataforma Android e iOS. Para sua instalação, é necessário habilitar a instalação de aplicativos nas lojas Play store e Apple store ou 
disponibilizado pelo serviço de saúde.

A Figura 1 ilustra a tela inicial do aplicativo com o Menu ativo. Esse Menu possui oito ícones que discorrem sobre a doença, tratamento, perguntas frequentes e a função do aplicativo.

Figura 1. Tela de Onboarding do aplicativo HemoCheck. Rio Verde/GO, 2020.

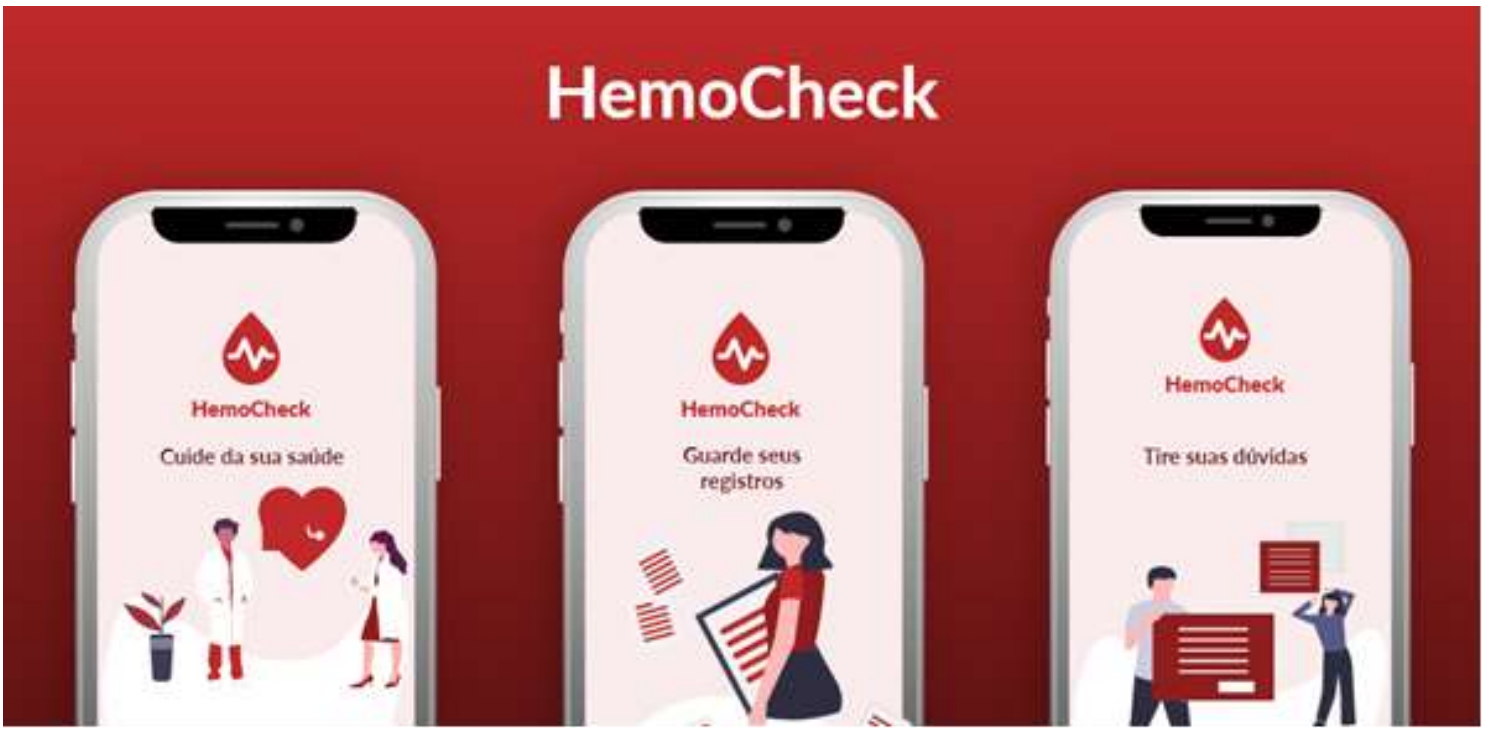

Fonte: Autores (2020).

Essa tela permite que usuário tenha uma breve introdução de algumas das funções do que o aplicativo HemoCheck é capaz de realizar, e em logo em seguida é possível que faça o seu cadastro e/ou login para que possa utilizá-lo.

A Figura 2 apresenta a tela do usuário. Essa página principal possui cinco opções, onde: uma tela fornece orientações sobre a doença, outra acerca do histórico de infusões, há uma página para o registro de intercorrência, uma tela de perfil do usuário e também um botão para ligar diretamente para o hemocentro que foi cadastrado pelo usuário.

Figura 2. Telas de Menu do Usuário do aplicativo HemoCheck. Rio Verde/GO, 2020.

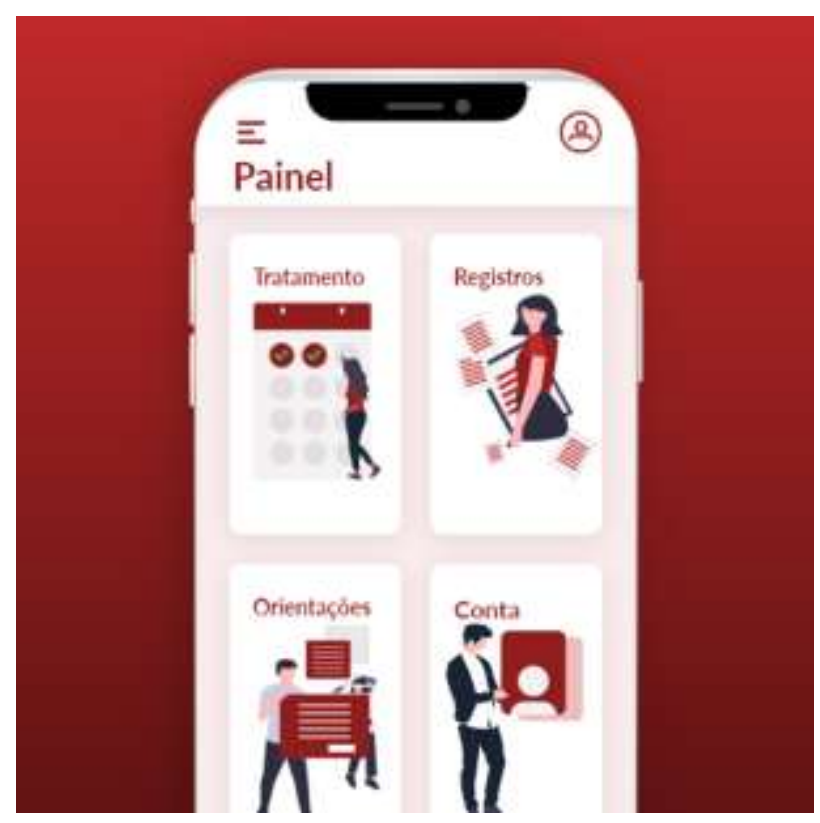

Fonte: Autores (2020) 
As telas de Menu do Usuário facilitam o entendimento do usuário sobre as possibilidades de atividades do aplicativo e como acessar as demais telas (com apenas um toque no item desejado), uma vez que dispõe de todas as funções em uma única tela, e conta com uma alta visibilidade e usabilidade.

As telas de cadastro de intercorrência possibilitam que o usuário coloque o horário e a data da infusão de fator, o local da aplicação, a dosagem e medicações utilizadas, se houve necessidade de ir ao hospital e se foi preciso alguma abordagem terapêutica de alta complexidade, como uma cirurgia. As telas estão disponíveis na Figura 3.

Figura 3. Telas de Cadastro de Intercorrências do aplicativo HemoCheck. Rio Verde/GO, 2020.

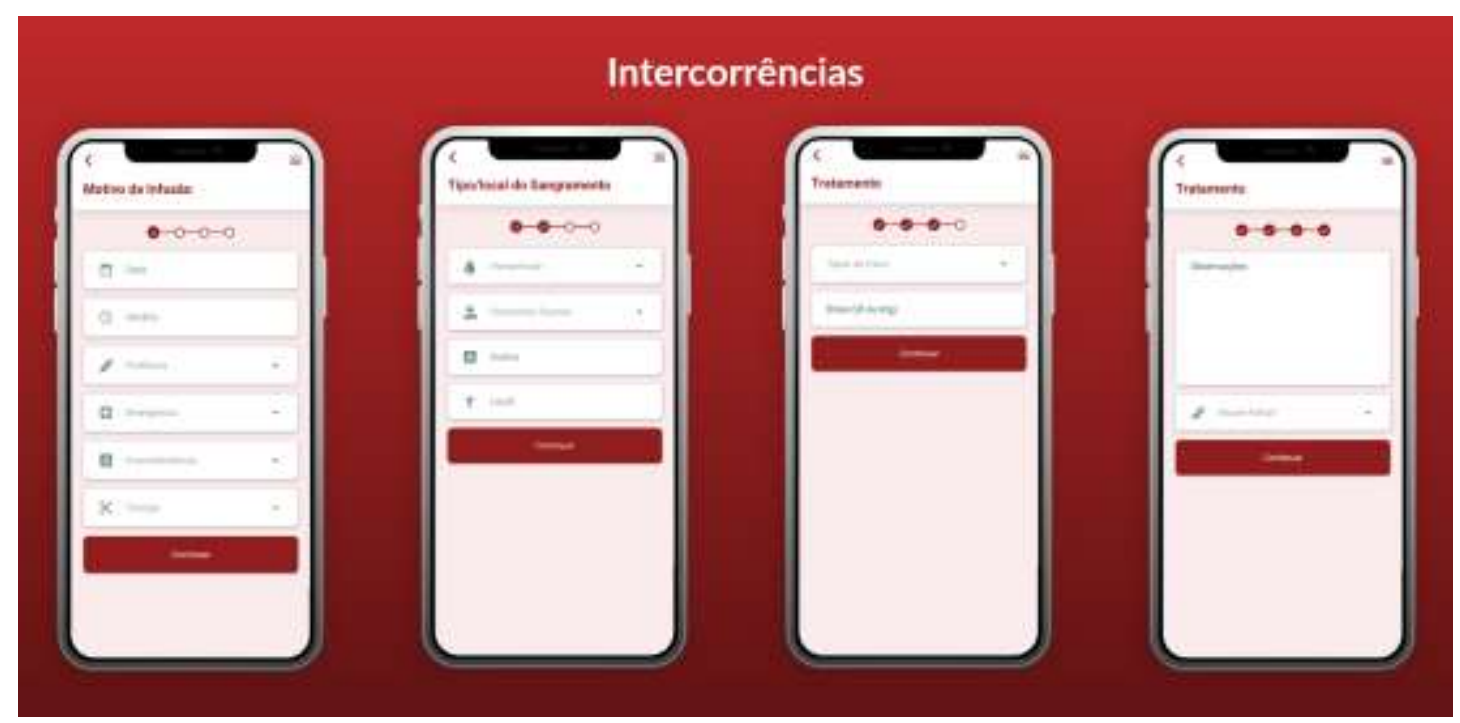

Fonte: Autores (2020).

As telas da Figura 3 são as que apresentam a principal função do aplicativo: o registro das infusões de fator. Como é possível verificar pela Figura, o aplicativo possui uma linguagem simples, e, é fácil de usar, desse modo, diversos usuários podem utilizá-lo e usufruir das funções.

A Figura 4 apresenta a quantidade de intercorrências, o local de infusão do fator, o horário que foi realizado, possibilita a exibição e remoção de cada infusão registrada. 
Figura 4. Telas com a Quantidade de Intercorrências do aplicativo HemoCheck. Rio Verde/GO, 2020.

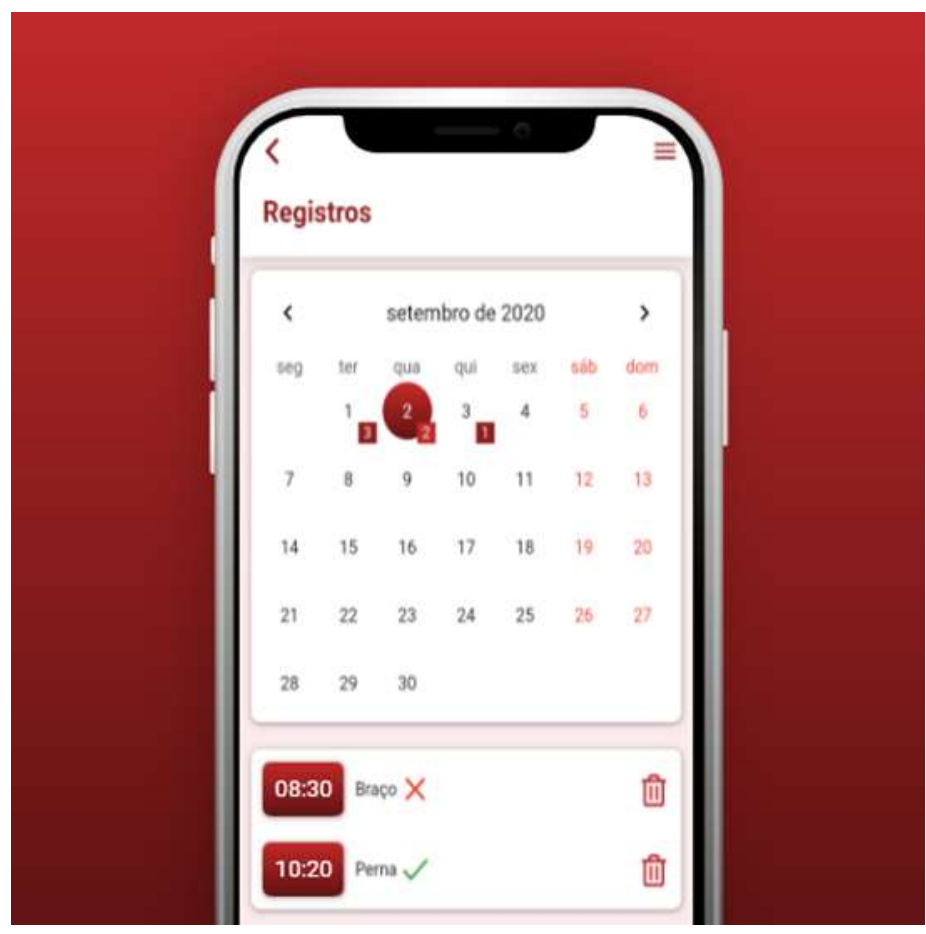

Fonte: Autores (2020)

Além disso, pode-se informar se a aplicação foi efetiva e eficaz ou não. Caso obtenha sucesso na administração do fator, no registro da tela de calendário, ao clicar na data irá aparecer a anotação realizada, o horário, o local, um ícone de "Check" (isso infere que a aplicação foi assertiva) e a lixeira para excluir o registro, se necessário. Em circunstâncias, quando a infusão não foi sucedida, um ícone de "X" surgirá na tela, ao lado das informações previamente citadas.

O usuário terá acesso a algumas orientações básicas acerca da dosagem do fator e medicações não recomendadas aos hemofílicos, pois, são anticoagulantes e podem ocasionar sangramentos espontâneos em pacientes com alto grau de severidade da hemofilia. As orientações estão disponíveis na Figura 5.

Figura 5. Telas de Orientações do aplicativo HemoCheck. Rio Verde/GO, 2020.

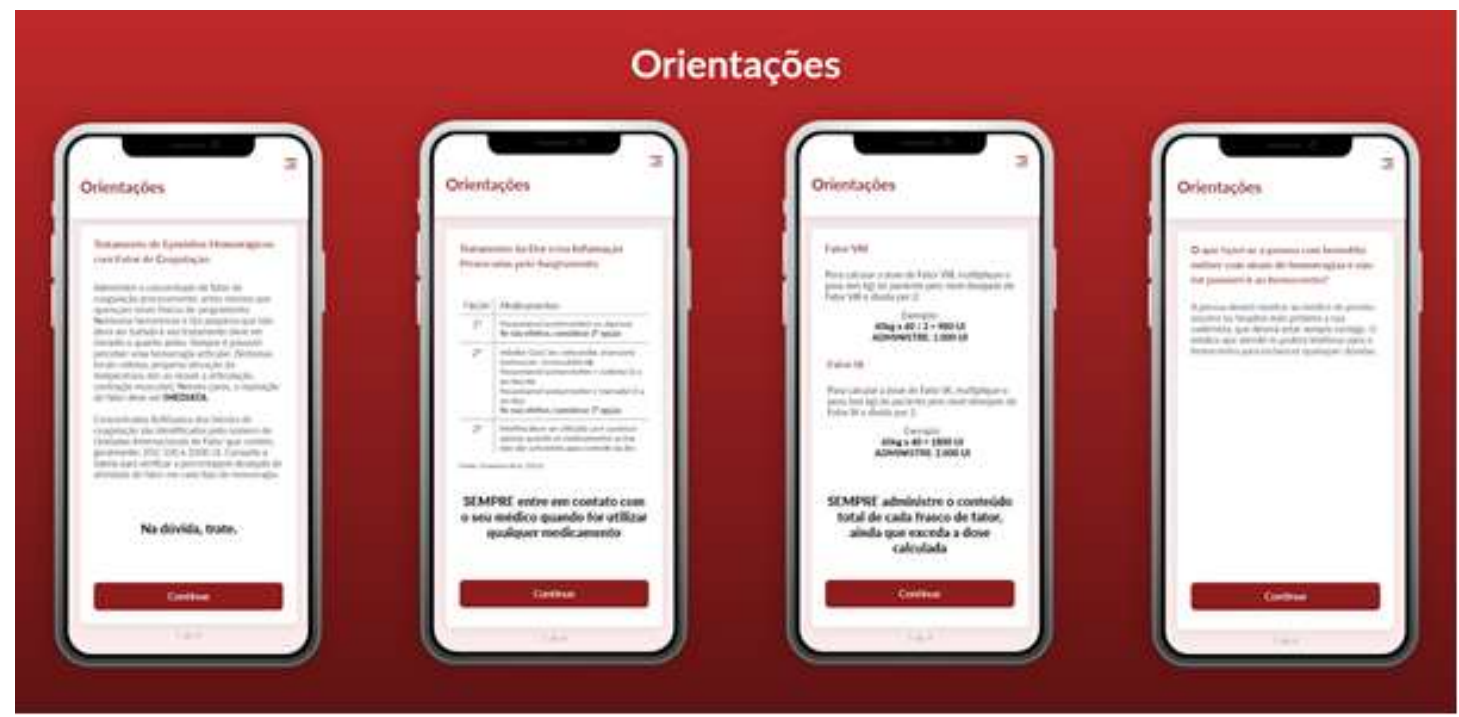

Fonte: Autores (2020). 
As orientações podem servir para casos de emergência para orientar quem desconhece a Hemofilia, como, informando quais medicações não podem ser administradas no paciente.

\section{Discussão}

O manejo ideal da hemofilia requer um monitoramento preciso e conformidade com o tratamento prescrito, porém, é um grande desafio para o paciente manter o controle desses dados usando o diário de infusão ou registros tradicionais no papel (Leone, 2011). Os aplicativos de saúde móveis e recursos de telessaúde podem aumentar o envolvimento e o autocuidado do paciente, principalmente se apresentarem login fácil, rastreamento em tempo real e exibição de dados simplificada. Estes recursos tornam o aplicativo mais intuitivo e fácil de usar (Baldwin, Singh, Sittig \& Giardina, 2017).

A cada dia que passa, os aplicativos móveis invadem a rotina dos profissionais de saúde e pacientes, sendo utililizados como meios para treinamentos, prevenção de doenças, monitoramento de doenças crônicas, ferramentas para avaliação de lesões e processos de enfermagem, entre outros (Souza et al., 2020; Oliveira, Vergara, Sampaio, \& Vasconcelos Filho, 2018; Lira, Rocha, Almeida, Amorim, \& Rocha, 2020).

Perante aos resultados encontrados, é possível verificar que o HemoCheck é um aplicativo simples, pensado e desenvolvido para facilitar os registros dos pacientes. Ao abrir a tela de Menu, ela já traz as funções primordiais do aplicativo, que são: o registro dos dados, orientações e anotações acerca do tratamento.

$\mathrm{O}$ crescente desenvolvimento tecnológico permite que esses registros sejam efetuados por meio de computadores e outros equipamentos eletrônicos. Os aplicativos, de maneira remota, que possibilitam o indivíduo registar quando tomou sua dose podem ser extremamente úteis e funcionais, principalmente, por permitir fácil acesso (Stirratt et al., 2015). O uso dos aplicativos móveis na área da saúde permite uma maior agilidade no fluxo de informações e interação entre os membros da equipe multiprofissional, e também, entre os profissionais e pacientes, vice-versa (Oliveira et al., 2021). De acordo com um ensaio clínico randomizado e controlado desenvolvido no Canadá com 41 hemofílicos, que comparou a adesão com a manutenção de registros de diários de papel com computadores portáteis mostrou que adesão ao registro do diário foi melhorada com o uso de computadores portáteis (Walker et al., 2004).

Os autorrelatos realizados por portadores de doenças crônicas são imperfeitos, mas apesar disso, são confiáveis (Stirrat et al., 2015). Em um estudo realizado no Hemocentro Regional de Juiz de Fora - Minas Gerais, observou-se que um indivíduo com $100 \%$ de adesão pela dispensação da farmácia, apresentou em seu diário, registros de infusões esporádicas (possivelmente por demanda) durante todo o mês, e infusões diárias apenas quando estava próximo da data de devolução dos frascos vazios ao Hemocentro (Ferreira, 2018).

A hemofilia é uma coagulopatia que exige a manutenção de bons registros de dados, principalmente no cuidado domiciliar, quando as infusões são realizadas na residência do hemofílico. Raramente, os profissionais de saúde utilizam as informações pessoais de cada paciente para melhorar o atendimento individual. Diante disso, os aplicativos de mHealth podem capacitar e amparar os pacientes (Khair \& Holland, 2014). A discussão sobre o diário de infusão, acerca do momento de dificuldade enfrentado e o fornecimento de um feedback sobre o tratamento, são essenciais para a adesão do paciente. Essa conversa entre profissional-paciente sobre o histórico de dosagem é 7,7\% mais eficaz do que a intervenção sem essa devolutiva (Demonceau et al., 2013).

Em um estudo retrospectivo realizado com uma amostra de 28 pacientes com hemofilia A grave e hemofilia B moderada em tratamento profilático, que avaliou o uso de um aplicativo móvel de monitoramento durante 6 meses, concluiu que a utilização do aplicativo de gerenciamento de terapia permitiu um aumento da adesão dos hemofílicos, um monitoramento dos medicamentos infundidos e das intercorrências ocorridas (Estelrich et al., 2018). 
Um estudo recente identificou que o registro de informações em meios eletrônicos oferecem mais vantagens do que o registro tradicional no papel, em especial, no que se refere à acessibilidade e disponibilidade de informações legíveis. Para aqueles pacientes que são contra o uso de aplicativos, cabe aos profissionais incentivar a introdução dessas tecnologias em longo prazo. É importante ressaltar que os profissionais e as campanhas de divulgação dos aplicativos não minimizem sua complexidade, porque os pacientes consideram a saúde algo complexo e acreditam que uma ferramenta simples não pode suprir a demanda, assim, acabam desconfiando da segurança e privacidade (Río-Lanza, Suárez-Vázquez, Suárez-Vázquez, \& Iglesias-Argüelles, 2020).

O aplicativo desenvolvido no presente estudo oferece benefícios ao Sistema Único de Saúde (SUS), como a melhoria da adesão dos pacientes em tratamento do Programa de Dose Domiciliar (DD), já que o resgistro se torna virtual e mais fácil de fazer, além de propiciar que todos os registros estejam sempre em mãos por meio do smartphone, assim, vai ser improvavél que o paciente esqueça de registrar a infusão ou de levar o diário no dia das consultas com especialista.

Todos os pacientes que fazem o tratamento em domicílio precisam fornecer diários ou planilhas de infusão. Nesses relatórios o paciente deverá fazer as anotações referentes aos episódios hemorrágicos e administração de concentrado de fator. Essas planilhas devem ser retornadas ao Centro de Tratamento de Hemofilia (CTH) para nova liberação de doses e registro apropriado (Brasil, 2015).

É essencial que posteriormente, sejam realizados estudos que abordem a avaliação dos usuários acerca do aplicativo HemoCheck, para assim, verificar sua eficácia em relação ao diário de infusão no formato de papel.

\section{Conclusão}

Para a construção do aplicativo foram elencados pontos fundamentais para assegurar uma ferramenta de educação em saúde efetiva e segura para os hemofílicos, considerando que seja uma estratégia eficaz para investir na qualidade de vida de pacientes crônicos pelo acompanhamento sistemático e rápido.

O desenvolvimento de um ambiente com o uso da linguagem dart e o framework flutter, revelou-se agradável de ser utilizado devido à documentação bem escrita, exemplos de aplicações para a área da saúde com essas tecnologias e bom apoio da comunidade, mesmo por serem relativamente recentes durante a realização deste trabalho.

Após o desenvolvimento do aplicativo, é possível afirmar que ele permite ao usuário registrar todas as informações das infusões, além de ter um calendário contendo todas as infusões já feitas. Foi criado um aplicativo móvel educativo simples, seguro e acessível para registrar os dados importantes dos pacientes com hemofilia e realizar o acompanhamento. Sendo assim, a pesquisa cumpriu seus objetivos e confirmou a hipótese prévia.

Destaca-se a necessidade de mensuração do impacto do aplicativo na saúde dos brasileiros, bem como a comparação dos custos na utilização da tecnologia móvel no acompanhamento e forma tradicional de consultas. Também é relevante a mensuração do impacto dessa tecnologia na vida desses pacientes e na sustentabilidade dos serviços de saúde.

Portanto, em trabalhos futuros devem ser desenvolvidas novas pesquisas para avaliar o uso do aplicativo criado, se é eficaz e resolutivo, a fim do seu aperfeiçoamento e de suprir a demanda dos usuários do HemoCheck. Também, se faz necessário a realização de mais produções acerca da temática para propiciar reflexões críticas atualizadas e pertinentes.

\section{Referências}

Baldwin, J. L., Singh, H., Sittig, D. F., \& Giardina, T. D. (2017). Patient portals and health apps: Pitfalls, promises, and what one might learn from the other. Healthc, 5 (3), 81-85. https://doi.org/10.1016/j.hjdsi.2016.08.004

Barca, D. A. A. V., Rezende, S. M., Simões, B. J., Pinheiro, K. N., Daisson, T., Sternick, G., Santo, M. L., \& Genovez, G. (2010). Hemovida Web Coagulopatias: um relato do seu processo de desenvolvimento e implantação. Cad. saúde colet., 18 (3). http://www.cadernos.iesc.ufrj.br/cadernos/images/csc/2010_3/artigos/CSCv18n3_pag434-44.pdf 
Bindhin, N. F., Freeman, B., \& Trevena, L. (2012). Pro-smoking apps for smartphones: the latest vehicle for the tobacco industry? Tobacco Control, 23. https://doi.org/10.1136/tobaccocontrol-2012-050598

Brasil. (2018). Hemofilia: conheça doença que afeta quase exclusivamente homens. Brasília: Ministério da Saúde. http://www.saude.gov.br/noticias/agenciasaude/46187-hemofilia-conheca-doenca-que-afeta-quase-exclusivamente-homens

Brasil. (2015). Manual de hemofilia. Brasília: Ministério da Saúde. http://bvsms.saude.gov.br/bvs/publicacoes/manual_hemofilia_2ed.pdf

Brasil. (2016). Manual de diagnóstico laboratorial das Coagulopatias Hereditárias e Plaquetopatias. Brasília: Ministério da Saúde. http://bvsms.saude.gov.br/bvs/publicacoes/manual_diagnostico_coagulopatias_hereditarias_plaqueopatias.pdf

Brasil. (2011). Manual de reabilitação na hemofilia. Brasília: Ministério da Saúde. http://bvsms.saude.gov.br/bvs/publicacoes/manual_reabilitacao_hemofilia.pdf

Brasil. (2017). Perfil das coagulopatias hereditárias no Brasil: 2015. Brasília: Ministério da Saúde. http://bvsms.saude.gov.br/bvs/publicacoes/perfil_coagulopatias_hereditarias_brasil_2015.pdf

Brasil. (2018). Perfil das coagulopatias hereditárias: 2016. Brasília: Ministério da Saúde http://bvsms.saude.gov.br/bvs/publicacoes/perfil_coagulopatias_hereditarias_2016.pdf

Burbeck, S. (1992). Applications programming in smalltalk-80 (tm): How to use model-view-controller (mvc). Smalltalk-80, 2, 1-11. http://stwww.cs.illinois.edu/users/smarch/st-docs/mvc.html

Cagnolati, D., Sankarankutty, A. K., Rocha, J. P. S., Beer, A., \& Silva, O. C. (2017). Hemostasia e Distúrbios da Coagulação. Rev. USP. https://sites.usp.br/dcdrp/wp-content/uploads/sites/273/2017/05/hemostasia_revisado.pdf

Demonceau, J., Ruppar, T., Kristanto, P., Hughes, D. A., Fargher, E., Kardas, P., Geest, S., Dobbels, F., Lewek, P., Urquhart, J., \& Vrijens, B. (2013). Identification and assessment of adherence-enhancing interventions in studies assessing medication adherence through electronically compiled drug dosing histories: a systematic literature review and meta-analysis. Drugs, 73(6), 545-62. <https://doi.org/10.1007/s40265-013-0041-3

Google Developers. (2020). Android Studio. https://developer.android.com/studio?hl=pt-br

Estelrich, M. M. S., Sánchez-Raga, J. M., Massanet, B. G., Delgado, O., Sampol, A., \& Canaro, M. (2018). Impact of Mhealth and Ehealth Applications on Hemophilia Treatment Management. Blood, 132. https://doi.org/10.1182/blood-2018-99-119139

Flutter. (2020). Home page. https://flutter.dev/

Federação Brasileira de Hemofilia [FBH]. (2020). O que é profilaxia? https://www.hemofiliabrasil.org.br/pagina/profilaxia

Ferreira, A. A. (2018). A Hemofilia: validação da versão brasileira do VERITAS-Pro para avaliação da adesão à profilaxia e custo-análise do tratamento. Tese de Pós-Graduação, Universidade Federal de Juiz de Fora, Juiz de Fora, MG, Brasil. http://repositorio.ufjf.br:8080/jspui/bitstream/ufjf/8289/1/adrianaaparecidaferreira.pdf

Free, C. J., Phillips, P., Galli, L., Watson, L., Felix, L., Edwards, P., Patel, V., \& Haines, A. (2013). The Effectiveness of Mobile-Health Technology-Based Health Behaviour Change or Disease Management Interventions for Health Care Consumers: A Systematic Review. PLOS Medicine, 10(1). https://doi.org/10.1371/journal.pmed.1001362

Fuck, M. P., \& Vilha, A. P. M. (2012). Inovação Tecnológica: da definição à ação. Revista Contemporâneos: Artes e Humanidades, 9, 1-21. https://www.revistacontemporaneos.com.br/n9/dossie/inovacao-tecnologica.pdf

IM, H., Song, J. Y., Cho, Y. K., Kim, Y. J., Kim, H. J., \& Kang, Y. J. (2013). The Use of Smartphone Applications in Stroke Rehabilitation in Korea. Brain Neurorehabil, 6(1), 33-40. https://doi.org/10.12786/bn.2013.6.1.33

Instituto Brasileiro de Geografia e Estatística [IBGE]. (2016). Acesso à internet e à televisão e posse de telefone móvel celular para uso pessoal. <https://www.ibge.gov.br/estatisticas/multidominio/condicoes-de-vida-desigualdade-e-pobreza/9171-pesquisa-nacional-por-amostra-de-domicilios-continuamensal.html?edicao $=28382 \& \mathrm{t}=$ downloads

Instituto Brasileiro de Geografia e Estatística [IBGE]. (2018). Acesso à internet e à televisão e posse de telefone móvel celular para uso pessoal. https://www.ibge.gov.br/estatisticas/multidominio/condicoes-de-vida-desigualdade-e-pobreza/9171-pesquisa-nacional-por-amostra-de-domicilios-continuamensal.html?edicao $=28382 \& \mathrm{t}=$ downloads

Khair, K., \& Holland, M. (2014). Managing hemophilia: the role of mobile technology. Smart Homecare Technology and TeleHealth, 2(39). https://doi.org/10.2147/SHTT.S40961

Leone, J. R. (2011). Utility of a wireless, handheld monitoring system in the management of hemophilia patients. Comput Inform Nurs, 29(9), 521-522. https://doi.org/10.1097/NCN.0b013e3182066356

Lira, T. B., Rocha, F. C. V., Almeida, C. A. P. L., Amorim, F. C. M., \& Rocha, L. P. V. (2020). Elaboração e avaliação de um protótipo-aplicativo para cuidador de idosos. Enfermeria Global, 19(3), 389-421. https://doi.org/10.6018/eglobal.396671

Mendez, C. B., Salum, N. C., Junkes, C., Almonte, L. N., \& Mendez, C. M. L. (2019). Aplicativo móvel educativo e de follow up para pacientes com doença arterial periférica. Rev. Latino-Am. Enfermagem, 27. http://www.scielo.br/scielo.php?script=sci_arttext\&pid=S0104-11692019000100306\&lng=en\&nrm=iso

Oliveira, A. R. F., \& Alencar, M. S. M. (2017). O uso de aplicativos de saúde para dispositivos móveis como fontes de informação e educação em saúde. RDBCI, 15(1), 234-245. https://doi.org/10.20396/rdbci.v15i1.8648137 
Oliveira, L. M. R., Vergara, C. M. A. C., Sampaio, H. A. C., \& Vasconcelos Filho, J. E. (2018). MHealth technology in the prevention and control of obesity from the perspective of health literacy: Lisa Obesidade. Saúde em Debate, 42(118), 714-723. https://dx.doi.org/10.1590/0103-1104201811814

Oliveira, M. C. P., Moura, A. K., Lima, K. M. O., Medeiros, M. C. W. C., Lira, M. N., \& Lima, J. R. (2021). Construção de um protótipo de aplicativo móvel para processo de enfermagem do paciente renal. Research, Society and Development, 10(3). http://dx.doi.org/10.33448/rsd-v10i3.13226

Organização para Cooperação e Desenvolvimento Econômico [OCDE]. (2002). Manual de Frascati. F-Iniciativas. http://www.ipdeletron.org.br/wwwroot/pdfpublicacoes/14/Manual_de_Frascati.pdf

Pereira, A. S., Shitsuka, D. M, Parreira, F. J., \& Shitsuka, R. (2018). Metodologia da pesquisa científica. UFSM. https://repositorio.ufsm.br/bitstream/handle/1/15824/Lic_Computacao_Metodologia-Pesquisa-Cientifica.pdf?sequence=1

Pôncio, T. G. H. O. (2018). Avaliação da qualidade de vida do portador de hemofilia e implementação do diário de infusão. Dis sertação de Mestrado, Universidade de São Paulo, Ribeirão Preto, SP, Brasil. https://www.teses.usp.br/teses/disponiveis/17/17155/tde-19072018102650/publico/THIARAGUIMARAESHELENODEOLIVEIRAPONCIOco.pdf

Portaria no 364 de 6 de maio de 2014. (2014). Saúde Legis.

Portaria no 478 de 16 de junho de 2014. (2014). Saúde Legis. http://bvsms.saude.gov.br/bvs/saudelegis/sas/2014/prt0478_16_06_2014.html

Río-Lanza, A. B., Suárez-Vázquez, A., Suárez-Vázquez, L., \& Iglesias-Argüelles, V. (2020). Mobile health (mhealth): facilitators and barriers of the intention of use in patients with chronic illnesses. Journal of Communication in Healthcare, 13(2), 138-146. https://doi.org/10.1080/17538068.2020.1777513

Sanders, W., \& Cumaranatunge, C. (2007). ActionScript 3.0 Design Patterns: Object Oriented Programming Techniques. O'Reilly Media Inc.

Souza, C. F. Q., Souza, S. M., Batista, R. P. S., Bandeira, T. P. M., Brandão Neto, W., \& Da Silva Junior, W. S. (2020). Aplicativo móvel como ferramenta de assistência e prevenção ao infarto agudo do miocárdio. Enfermería Actual de Costa Rica, (39), 129-143. https://dx.doi.org/10.15517/revenf.v0i39.41072

Stirratt, M. J., Jacob, J. D., Crane, H. M., Simoni, J. M., Czajkowski, S., Hilliard, M. E., Aikens, J. E., Hunter, C. M., Velligan, D. I., Huntley, K., Ogedegbe, G., Rand, C. S., Schron, E., \& Nilsen, W. J. (2015). Self-report measures of medication adherence behavior: recommendations on optimal use. Translational behavioral medicine, 5, 470-482. https://doi.org/10.1007/s13142-015-0315-2

Tibes, C. M. S., Dias, J. D., \& Zem-Mascarenha, S. H. (2014). Aplicativos móveis desenvolvidos para a área da saúde no Brasil: revisão integrativa da literatura. Rev Min Enferm., 18(2), 471-78. https://doi.org/10.5935/1415-2762.20140035

Visual Studio Code. (2020). Home page. https://code.visualstudio.com/

Zazo, M. A., Falcão, R. P., \& Pasquini, R. (2013). Tratado de Hematologia. Editora Atheneu, 627-635.

Walker, I., Sigouin, C., Sek, J., Almonte, T., Carruthers, J., Chan, A., Pai, M., \& Heddle, N. (2004). Comparing hand-held computers and paper diaries for haemophilia home therapy: a randomized trial. Haemophilia, 10(6), 698-704. https://doi.org/10.1111/j.1365-2516.2004.01046.x.

World Health Organization [WHO]. (2011). mHealth New horizons for health through mobile technologies. Global Observatory for eHealth series, 3. https://www.who.int/goe/publications/goe_mhealth_web.pdf

World Health Organization [WHO]. (2012). Guidelines for the management of Hemophilia. Blackwell Publishing in Haemophilia, 6. http://www1.wfh.org/publications/files/pdf-1472.pdf 